\title{
Satisfacción de estudiantes de secundaria nocturna y su incidencia en el abandono escolar
}

\author{
Students' Satisfaction at Night High School and Its Influence in School Dropout
}

\section{Satisfação dos estudantes do ensino médio noturno e seu impacto sobre a evasão escolar}

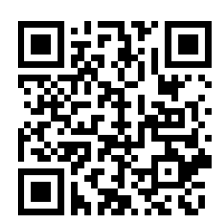

José Laurian Ramírez-Díaz

Ministerio de Educación Pública

Cartago, Costa Rica

jramirez@ucatolica.ac.cr

Fiorella Hidalgo-Solano²

Universidad Católica de Costa Rica

San José, Costa Rica

fiorella-h93@hotmail.com

Recibido • Received • Recebido: 03 / 06 / 2016

Corregido $\cdot$ Revised $\cdot$ Revisado: $02 / 11 / 2017$

Aceptado • Accepted • Aprovado: $05 / 12$ / 2017

\begin{abstract}
Resumen: Este documento presenta un estudio que analizó dimensiones referentes a la satisfacción que muestran estudiantes en secundaria nocturna para con su proceso educativo, a partir de la valoración que tienen de su propia motivación, las instalaciones físicas y las interacciones que se dan entre estudiantes y el profesorado, específicamente en ámbitos de educación técnico profesional. Esto, con el fin de analizar componentes que aumenten el sentimiento de bienestar y potenciar la promoción y el éxito académico con base en criterios preventivos del abandono escolar. Para lo anterior se realizó un análisis factorial con información obtenida de un cuestionario basado en una escala de Likert aplicado a una muestra de 120 participantes, con lo cual se logró identificar algunos indicadores importantes para considerar en acciones de prevención del abandono escolar en poblaciones estudiantiles de personas adultas. La información recabada se presenta en tablas que exponen los indicadores sobre satisfacción de estudiantes en infraestructura, trato docente y motivación.
\end{abstract}

Palabras claves: Satisfacción; infraestructura; interacción; docencia; secundaria.

\footnotetext{
${ }^{1}$ Magíster en Tecnología Educativa por la Universidad Estatal a Distancia. Licenciado y Bachiller en Orientación por la Universidad Católica de Costa Rica y la Universidad de Costa Rica respectivamente. Bachiller en Psicología por la Universidad Católica de Costa Rica. Diplomado en TIC y Educación por la Organización de Estados Iberoamericanos.

${ }^{2}$ Bachiller en Psicología por la Universidad Católica de Costa Rica. Actualmente cursa el programa de licenciatura.
} 
doi: http://dx.doi.org/10.15359/ree.22-1.14

URL: http://www.una.ac.cr/educare

CORREO: educare@una.cr

\begin{abstract}
This paper presents an analysis on the satisfaction of night school students about their educational process, from the valuation they have of their own motivation, physical infrastructure facilities, and interpersonal relationships between students and teachers, specifically in the technical and professional education fields. All this aimed to analyze components that increase the feeling of well-being, and enhance the promotion and academic success based on criteria to prevent the school dropout. To do so, we conducted a factor analysis with information obtained from a questionnaire based on a Likert scale. The questionnaire was applied to a sample of 120 participants; with it, we identified some important indicators to be considered in actions to prevent dropout in adult students. The collected information was presented in statistical tables, which display indicators of satisfaction about infrastructure, teacher's treatment, and student's motivation.
\end{abstract}

Keywords: Satisfaction; infrastructure; interaction; teaching; high school.

Resumo: Este trabalho apresenta um estudo que analisou dimensões relacionadas com a satisfação demostrada por estudantes do ensino médio noturno, sobre seu processo educacional, a partir da avaliação que eles têm da sua própria motivação, instalações físicas e interações que ocorrem entre estudantes e professores, especificamente nas áreas do ensino técnico profissional. Este com a finalidade de analisar os componentes que aumentam a sensação de bem - estar e melhorar a promoção e sucesso acadêmico, com base em critérios de prevenção da evasão escolar. Para lograr nosso propósito, realizou-se uma análise dos fatores a partir de informações obtidas de um questionário, fundamentado em uma escala de Likert, aplicado a um grupo de 120 participantes. Com isso se logrou identificar alguns indicadores importantes a considerar na prevenção da evasão escolar em populações de estudantes adultos. As informações recolhidas são apresentadas em tabelas que mostram indicadores de satisfação dos estudantes relacionados com a infraestrutura, tratamento e motivação dos professores.

Palavras-chave: Satisfação; infraestrutura; interação; ensino; secundário.

\title{
Introducción
}

Sobre el abandono escolar han sido muchas las investigaciones que se han dado tanto en el nivel internacional como en el país y, entre las causas más frecuentes que se relacionan con este fenómeno se encuentran el nivel socioeconómico del estudiantado y la limitación que en algunos casos se tiene para integrarlo activamente a los procesos educativos, considerada aquí la formación como un proceso comunitario y social (Rojas, 2000). En el caso de las instituciones de secundaria nocturna tanto de educación técnica como académicas, las condiciones de la población estudiantil varían en forma destacada respecto a estudiantes de colegios diurnos, donde destacan componentes como la edad, los subsidios para estudiar como las becas, cuyos requisitos tienden a restringir su alcance hacia poblaciones de personas adultas por las propias características de la población de estudiantes, entre otros más. 
En Costa Rica, los estudios sobre abandono escolar plantean la desmotivación estudiantil relacionada a problemas socioeconómicos, bajo rendimiento académico, ausentismo y problemas de conducta; el perfil de quien abandona el colegio describe a una persona sin motivación, con carencias distintas y sin mayor estímulo en su familia (Jiménez y Gaete, 2010). Esto puede acrecentarse en los colegios nocturnos donde la mayoría de sus estudiantes son sujetos adultos que ocasionalmente presentan una necesidad de trabajar para afrontar sus necesidades básicas, de modo que la formación les implica una forma de optimizar su condición laboral u obtener alguna certificación para acceder a trabajos mejor remunerados. Así mismo, con base en las investigaciones realizadas en el país, uno de los agentes de mayor protagonismo preventorio sobre el fenómeno implica la motivación y la mística del personal docente que atiende al estudiante en las aulas (Programa Estado de la Nación, 2015).

Sin embargo, la mayoría de estudios sobre abandono escolar en secundaria considera un protagonismo de la persona adolescente, el mayor número de la población activa en este sentido pertenece a este grupo etario. No obstante, las secciones técnicas y los colegios académicos de horario nocturno proponen una oportunidad inmejorable para muchas personas que, por diferentes razones, no concluyeron sus estudios o requieren mejorarlos. Es relevante, así, otorgar una voz al estudiantado adulto, de modo que puedan conocerse sus necesidades, su percepción del servicio que se le brinda, cuáles elementos del proyecto de vida inciden en la toma de decisiones sobre su formación, qué previene el abandono de las aulas y, la realidad que afronta en cada jornada de estudio, entre otros más.

Por lo anterior, la presente investigación propuso un análisis de elementos que previenen la deserción en tres dimensiones, con base en la percepción de dicentes de una institución técnica nocturna: la automotivación hacia el estudio, la infraestructura donde reciben lecciones y el trato docente.

\section{Marco teórico}

La satisfacción puede definirse desde varias aristas. Una de ellas parte de la dimensión personal, la cual se relaciona con la valoración individual de la calidad de vida, regida por apreciaciones subjetivas y objetivas, que trascienden lo económico (Cardona y Agudelo, 2007). Además, guarda estrecha relación con el bienestar personal o el bienestar subjetivo, analizado por la psicología a partir de juicios cognitivos y respuestas emocionales (Gutiérrez y Gonçalves, 2013).

El bienestar subjetivo es un constructo basado en subjetividades, pero analizado y medido correlacionalmente con factores objetivos, es decir, la satisfacción de las necesidades básicas (Peña y Sánchez, 2009), que se relaciona con la calidad de vida, un concepto surgido en Estados Unidos a mitad del siglo anterior, con el fin de evaluar la percepción de la vida y seguridad económica de los sujetos (Urzúa y Caqueo-Urízar, 2012). La calidad de vida considera los cambios sociales 
positivos que incluyen elementos psicosociales como evaluaciones, expectativas, aspiraciones, percepción y el entorno (Ayuste et al., 2001 en Luna, 2012; Casas, 1996).

En contextos educativos, la satisfacción se relaciona con los componentes analizados anteriormente y su estudio deriva de los mismos escenarios. En el caso de la satisfacción de estudiantes, es referida por Zas (2002, en Jiménez, Terriquez y Robles, 2011) como un elemento primordial de la calidad de la educación, y uno de los indicadores más relevantes para medir la calidad de los procesos de enseñanza. La satisfacción escolar parece estar relacionada directamente con el rendimiento académico (Abarca, Cáceres, Jiménez, Moraleda y Romero, 2013). Esto se traduce en la relevancia de analizar los componentes relacionados con la satisfacción que muestra el estudiantado respecto a la infraestructura del centro educativo, el trato de docentes, así como la automotivación para concluir sus proyectos de formación en la secundaria nocturna. El colegio se propone como un factor de motivación circunscrito al proyecto de vida o, caso contrario, como un componente que limita o dificulta el adecuado desarrollo del sujeto en este sentido (Edel, 2003).

Es importante rescatar que, por rendimiento académico, se comprende un fenómeno multicausal, incidido por aspectos internos y externos al sujeto (Garbanzo, 2007; GonzálezPienda, 2003). El rendimiento académico es considerado desde una perspectiva cuantitativa (pruebas educativas) y cualitativa (valoración subjetiva de los resultados). El rendimiento académico es considerado como producto del sistema educativo, compuesto por diferentes dimensiones, tal como se explicó anteriormente (Montes y Lerner, 2011). En este sentido, se pueden plantear las variables del estudio con base en componentes internos y externos, tal como se define operacionalmente en la Tabla 1.

Tabla 1: Componentes internos y externos relacionados con el rendimiento académico

\begin{tabular}{|c|c|c|}
\hline Componente & Definición literal & Definición operacional \\
\hline Infraestructura & $\begin{array}{l}\text { Bienes muebles como mobiliario, equipo e } \\
\text { inmuebles (terrenos) que utilizan estudiantes y } \\
\text { docentes en los procesos educativos (Sáenz, 2004). }\end{array}$ & $\begin{array}{l}\text { Estado de la infraestructura del centro } \\
\text { educativo y condiciones para favorecer el } \\
\text { estudio }\end{array}$ \\
\hline Trato docente & $\begin{array}{l}\text { Proceso de interacción bidireccional donde } \\
\text { docentes y estudiantes se influyen, exteriorizan } \\
\text { e internalizan sus puntos de vista y construyen } \\
\text { nuevas realidades (Bertoglia, 2005). }\end{array}$ & Interacción entre docentes y estudiantes \\
\hline Automotivación & $\begin{array}{l}\text { "La disposición positiva para aprender y continuar } \\
\text { haciéndolo de una forma autónoma" (Ajello, en } \\
\text { Naranjo, 2009, p. 153). }\end{array}$ & Deseos de superación del estudiantado \\
\hline
\end{tabular}

Nota: Elaboración propia. 
En general, estos componentes (infraestructura, trato docente y automotivación) se relacionan con el estado de bienestar estudiantil, de modo que pueden favorecer su permanencia en el colegio o, caso contrario, el abandono escolar. Así mismo, el disfrute subjetivo de la vida y de todas las actividades relacionadas es un componente de importante consideración por parte de la psicología, de modo que podría suponerse una relación directa entre la satisfacción entre estos y la conclusión del proyecto formativo de cada dicente. En el ámbito educativo, por tanto, la formación implica la humanización de los procesos de aprendizaje, como un objetivo propuesto para prevenir la deserción y coadyuvar la permanencia del estudiantado en el sistema educativo (León, 2011).

Los centros educativos constituyen una realidad social que, con el paso del tiempo, se han establecido como depositarios de la transmisión, reconstrucción y desarrollo del conocimiento (Teixedó, 2005). Para un adecuado funcionamiento, cada centro educativo debe contar con una infraestructura, tal como lo explicó Municio (citado por Gellnier, 1967) décadas atrás: "cada centro necesita su estructura para responder a sus objetivos, como las estructuras de las casas cambian con el clima, con la cultura o el paisaje" (p. 95).

La infraestructura, en el ámbito propiamente educativo, puede considerarse como el espacio donde los sujetos y los grupos se relacionan entre sí, de modo que forma parte de los componentes que constituyen los contextos formativos y, por tanto, de la socialización, en tanto contribuye a la identificación de estos con su centro de estudios (Sáenz, 2004). En este sentido, Brea (2014) explica que "la identidad de lugar incluye un sentido de pertenencia, enraizamiento, normas y valores acerca del espacio común y del espacio personal. La identidad social y la identidad de lugar están íntimamente relacionadas" (p. 21).

Así las cosas, el sentido de pertenencia se plantea a partir de un grupo o una comunidad o, dicho en otras palabras, el significado de ser miembro (Flores, 2005). Se formula, entonces, que la infraestructura de los centros educativos se relaciona con el favorecimiento del sentido depertenencia a un grupo específico y, por tanto, al centro de estudio como un todo, donde se dan relaciones interpersonales entre estudiantes y profesorado como parte del proceso. Además, promueve la permanencia del estudiantado en las aulas o, dicho en otra forma, previene la deserción.

La deserción, descrita por el Ministerio de Educación Pública como abandono escolar o inasistencia, históricamente ha presentado los mayores índices en los sistemas académicos nocturnos y en la educación técnica nocturna, en primero y segundo lugar respectivamente (Jiménez y Gaete, 2010). La deserción del sistema educativo tiene, como mayor consecuencia, la privación de las poblaciones más vulnerables de posibilidades para su vida futura y obstruye oportunidades de superar la pobreza y merma las posibilidades de movilidad social propuesta por la educación (Herrera, 2012). Sin embargo, un elemento que se considera como uno de los mayores preventorios de la deserción trata sobre el impacto del profesorado en sus estudiantes (Fallas y Ross, 2014), una situación que respalda el Quinto Informe del Estado de la Educación 
doi: http://dx.doi.org/10.15359/ree.22-1.14

URL: http://www.una.ac.cr/educare

CORREO: educare@una.cr

(Programa Estado de la Nación, 2015, p. 141) al explicar que "una vez que los niños ingresan a la escuela, ningún otro factor es tan importante como la calidad de los docentes", de modo que es sustancial potenciar este elemento. y valorar su incidencia precisamente en los contextos donde se presenta el mayor índice de deserción a nivel nacional.

Es importante mencionar que el estudiantado alcanza mayores niveles de motivación con experiencias calidad en el aula respecto a la cantidad, por lo cual el profesorado debe procurar la automotivación en cada dicente para con su proceso formativo (Navarrete, 2009). Se propone, así, que el personal docente plantee tres objetivos de motivación: primeramente la promoción del interés, segundo el dirigir y mantener el esfuerzo colectivo y, tercero, lograr el objetivo de aprendizaje fijado con anterioridad (Mingorance, 2010).

Es importante considerar que el Quinto Informe del Estado de la Educación (Programa Estado de la Nación, 2015) explica que, a nivel latinoamericano, Costa Rica se ubicaba entre las naciones con mayor equidad educativa en poblaciones de 21 a 30 años en 1990, pero actualmente se sitúa en niveles comparables al resto de naciones centroamericanas, lo cual se puede considerar como un retroceso, por lo que se propone como un fenómeno que afecta la motivación del estudiantado.

Respecto a la motivación, puede suponerse como un proceso de seguimiento, mantenimiento y regulación de actos, que producen cambios ambientales y que coinciden con limitaciones individuales (Soriano, 2001). Ajello (2003, en Naranjo, 2009, p. 153) explica que "en el plano educativo, la motivación debe ser considerada como la disposición positiva para aprender y continuar haciéndolo de una forma autónoma". En un sentido más individualista, la motivación es el deseo o interés por alguna actividad que brota a lo interno del mismo individuo, la cual podría definirse como automotivación.

En el entorno educativo podría explicarse que la automotivación "tiene como objetivo la experimentación de la autorrealización, por el logro de la meta, movido especialmente por la curiosidad y el descubrimiento de lo nuevo"(Ospina, 2006, p. 159). En este sentido, es importante diferenciar la motivación extrínseca y la intrínseca: la primera responde al entorno y la segunda parte del propio individuo, de su iniciativa e intereses (Baracho, 2010). La automotivación está referida en especial a la clasificación intrínseca.

Con base a lo anterior, se planteó como objetivo general de investigación: Analizar la satisfacción de estudiantes de secundaria nocturna en dimensiones de infraestructura, trato docente y automotivación. Para ello se propusieron tres objetivos específicos: (1) Identificar algunas características relevantes de la infraestructura del centro educativo que motivan a sus estudiantes para continuar sus estudios; (2) Sintetizar las principales dinámicas que median en el trato del personal docente hacia estudiantes que plantean criterios de motivación con el estudio y (3) Describir algunos componentes de automotivación presentes en estudiantes de educación secundaria nocturna, respecto a la continuación de sus estudios.

\section{6} José Laurian Ramírez-Díaz y Fiorella Hidalgo-Solano

Los artículos de la Revista Electrónica Educare del Centro de Investigación y Docencia en Educación de la Universidad Nacional, Costa Rica, se comparten bajo términos de la Licencia Creative Commons: Reconocimiento, № Comercial, Sin Obra Derivada 3.0 Costa Rica. Las autorizaciones adicionales a las aquí delimitadas se pueden obtener en el correo: educare@una.cr 


\section{Metodología}

El estudio se basó en un modelo cuantitativo de diseño explicativo, ya que buscó analizar la relación de componentes que inciden en la satisfacción de estudiantes de secundaria nocturna (Müggenburg y Pérez, 2007), referidos inicialmente a infraestructura, el trato docente y la motivación de estudiantes de secundaria técnica nocturna. La muestra se estableció en un total de 120 sujetos elegidos al azar, que en su mayoría se ubicaron en el estadio de desarrollo de adultez emergente (19 a 25 años) y adultez joven (26 a 40 años) según Papalia, Duskin y Martorell (2012) (véase Tabla 2).

Tabla 2: Muestra del estudio distribuida por género y estadio del desarrollo, según valores absolutos

\begin{tabular}{lcccc}
\hline & Persona adulta emergente & Persona adulta joven & Persona adulta intermedia & Total \\
\hline Hombre & 35 & 8 & 1 & 44 \\
Mujer & 48 & 27 & 1 & 76 \\
\hline Total & 83 & 35 & 2 & 120 \\
\hline
\end{tabular}

Nota: Elaboración propia.

La institución donde se realizó el estudio está ubicada en el cantón La Unión, Cartago. Con una modalidad técnica nocturna, su matrícula inicial para 2015 fue de 326 estudiantes, 306 en 2016 y 297 en 2017. Nueve de cada diez estudiantes son bachilleres en secundaria. Las edades oscilan entre los 19 a los 23 años, y la relación por género es de cuatro mujeres por cada seis varones. Más de la mitad de la población realiza alguna actividad remunerada, y ocho de cada diez estudiantes es soltero o soltera. Además, un individuo de cada cinco es padre o madre, en su mayoría de un solo hijo o hija, y provienen de sistemas familiares de entre cuatro a siete personas (Ramírez, 2015).

El instrumento para recabar la información constó de un cuestionario de 38 reactivos sobre una escala de Likert, distribuida en una escala de valoración de entre 1 (Total Desacuerdo) a 5 (Totalmente de acuerdo), que fue analizado con el software Statistical Package for the Social Sciences (SPSS). Del análisis factorial y el análisis de fiabilidad se determinaron los reactivos que proponen una valoración adecuada de los componentes que involucraron el estudio propuesto inicialmente, sobre los cuales se desarrolló el análisis final, presente en este documento.

\section{Resultados, análisis y discusión}

Con base en el análisis factorial, se redujo el estudio a dos dimensiones. Inicialmente se propusieron los componentes de infraestructura, trato docente y motivación, pero el resultado final de las dimensiones dio dos básicas, las cuales se derivan como se explica a continuación: 
Motivación: relacionada con la automotivación planteada originalmente; pero que, posterior al análisis factorial, se ha ampliado su consideración a componentes de infraestructura y estímulo docente.

Trato docente: una de las dimensiones tal cual se planteó inicialmente, y que está relacionada con la incidencia que tiene el profesorado sobre la población estudiantil.

Motivación: esta dimensión se relaciona con la ubicación del colegio, espacio de las aulas y ayuda docente hacia el estudiantado, que les motiva para continuar sus estudios. Particularmente se considera que esos componentes se unen en una sola dimensión ya que, tal como se indicó anteriormente, la automotivación tiene factores internos y externos. Sobre esta dimensión es importante considerar la incidencia que tiene la repitencia en la historia académica de la muestra, ya que más de la mitad de participantes ha presentado esta condición en algún momento durante su paso por la secundaria (véase Tabla 3).

Tabla 3: Repitencia

\begin{tabular}{lcc}
\hline & Frecuencia & Porcentaje \\
\hline Sí & 63 & 52.5 \\
No & 57 & 47.5 \\
\hline Total & 120 & 100.0 \\
\hline
\end{tabular}

Nota: Elaboración propia.

En este sentido, es importante considerar que aunque la repitencia puede ser un factor común entre la muestra al implicar a más de la mitad de esta y, que entre algunas de sus implicaciones se plantea el atraso en el cumplimiento del proyecto de formacióny, eventualmente, sentimientos de frustración y otros derivados, existen distintos elementos que proponen un bienestar subjetivo como constructo basado en subjetividades que, al final, plantean la satisfacción de las necesidades básicas (Peña y Sánchez, 2009). La formación para una ocupación propone una intencionalidad implícita de mejorar la calidad de vida, lo cual formula un motivo para continuar en el sistema educativo y concluir las metas de formación para el estudiantado. También, la infraestructura plantea un componente importante para la identificación con el centro de estudios y la conclusión del proyecto de formación. Al respecto, Brea (2014) explica que el sentido de pertenencia se relaciona con la identidad social y la identidad individual.

Es importante considerar la valoración general de esta dimensión de motivación, según la información referida por la muestra. En la Tabla 4 se expone la percepción general del desempeño de los componentes de ubicación, espacio de las aulas, el interés promovido por el personal docente para el estudio, y la responsabilidad que muestra para impartir sus lecciones, según la programación anual establecida.

8 José Laurian Ramírez-Díaz y Fiorella Hidalgo-Solano

Los artículos de la Revista Electrónica Educare del Centro de Investigación y Docencia en Educación de la Universidad Nacional, Costa Rica, se comparten bajo términos de la Licencia Creative Commons: Reconocimiento, № Comercial, Sin Obra Derivada 3.0 Costa Rica. Las autorizaciones adicionales a las aquí delimitadas se pueden obtener en el correo: educare@una.cr 
Tabla 4: Valoración general de los componentes de motivación para el estudio

\begin{tabular}{lcc}
\hline & Frecuencia & Porcentaje \\
\hline Ineficiente & 5 & 4,2 \\
Baja & 66 & 55,0 \\
Bueno & 23 & 19,2 \\
Óptimo & 26 & 21,7 \\
\hline Total & 120 & 100,0 \\
\hline
\end{tabular}

Nota: Elaboración propia.

En este sentido, es substancial aclarar que más de la mitad de participantes consideran que esta dimensión debe mejorar. Si bien la motivación tiene un componente intrínseco importante, tal como se analizó anteriormente, la incidencia de la infraestructura y la motivación docente son importantes para el estudiantado. Las mejoras que se proponen se refieren, entonces, a un mayor cumplimiento del programa establecido, impartir lecciones con base en la cantidad determinada, y motivar más para concluir el proceso de formación. Por otra parte, se demanda una mejor infraestructura, considerando el estado de los inmuebles y los recursos como mesas y sillas, pues las facilidades de acceso se pueden mejorar a través de un eficiente servicio de transporte público, con horarios de estudio que se articulen adecuadamente.

La segunda dimensión corresponde al trato docente. Los elementos considerados trataron sobre el aprovechamiento del tiempo, la metodología docente y lo agradable que es, para el estudiantado, la calidad en general del profesorado y la preocupación de este por el bienestar de sus dicentes. La Tabla 5 expone la valoración general del trato docente y la satisfacción estudiantil con el proceso de formación, propone también un área de mejora, según la percepción de la muestra. Tal como se mencionó anteriormente, ningún otro factor parece tener la relevancia en los procesos de formación, tal como la calidad de sus docentes (Programa Estado de la Nación, 2015).

Tabla 5: Valoración general del trato docente

\begin{tabular}{lcc}
\hline & Frecuencia & Porcentaje \\
\hline Ineficiente & 17 & 14,2 \\
Bajo & 76 & 63,3 \\
Bueno & 12 & 10,0 \\
Óptimo & 15 & 12,5 \\
\hline Total & 120 & 100,0 \\
\hline
\end{tabular}

Nota: Cuestionario aplicado en la investigación.. 
doi: http://dx.doi.org/10.15359/ree.22-1.14

URL: http://www.una.ac.cr/educare

CORREO: educare@una.cr

Quienes escriben consideran que la calidad docente promueve sentido de seguridad en el estudiantado, sentimientos referidos a la confortabilidad $y$, también, tal como se ha explicado anteriormente, incentivan la finalización de los estudios como una estrategia para mejorar la calidad de vida y de realización personal. Es importante considerar estrategias de mejora en maximización del tiempo de lecciones, las metodologías planteadas para dar la lección, la calidad general del profesorado y la preocupación de este por el bienestar general de sus estudiantes. Una de las estrategias que puede emplearse, en este sentido, es mejorar los procesos de interacción, donde se exterioricen puntos de vista tanto de estudiantes como docentes, y valorar situaciones reales con base en estrategias de aprendizaje, que consideren situaciones cotidianas, con el fin de mejorar la percepción del estudiantado sobre la calidad en el trato docente (Bertoglia, 2005). Así mismo, es importante valorar, a futuro, otras dimensiones que puedan constituirse en factores que coadyuven a las personas en la culminación de sus proyectos de estudio, ya que en este caso solo se valoraron algunas.

\section{Conclusiones}

El personal docente, tal como se explicó, parece ser un componente esencial que incide sobre la satisfacción estudiantil en los procesos formativos $y$, por tanto, en la motivación para el estudio y su permanencia en las aulas. Es interesante que, aun cuando no existe una valoración óptima sobre el desempeño respecto a su gestión, el estudiantado mantiene en firme su permanencia en el sistema educativo formal, lo cual hace pensar que, de mejorar esta dimensión, el abandono escolar podría disminuir, no solo en contextos de educación nocturna, sino a nivel general. Los elementos que motivan al alumnado para mantenerse estudiando podrían ser varios, pero muy posiblemente, estén relacionados con la motivación que les genera el cumplimiento de metas de formación, para el mejoramiento de las condiciones de vida. En este sentido, fue interesante percibir, en este estudio, que parece existir una relación directa en la percepción del estudiantado entre el profesorado y el salón de clase, lo cual podría explicar por qué, aunque las condiciones de infraestructura no sean las más adecuadas, el trato docente parece ser más importante y, por tanto, ser un elemento que motive la continuación en las aulas, tal cual plantean otros estudios mencionados en este texto.

Aunado a lo anterior, es importante considerar que la motivación no solo debe considerar al grupo de estudiantes, sino también al profesorado, tanto para sí mismo, así como en su interacción con sus dicentes. En este sentido, se tiene claro que, en el salón de clase, la interacción se da entre estudiantes y docentes, lo cual conlleva una realidad en que las necesidades humanas son básicamente las mismas para ambos grupos. Es importante, por tanto, emplear estrategias de motivación que beneficien al personal docente para que, de esta forma, repercuta positivamente en el estudiantado. Es muy posible que la idoneidad del espacio donde se reciben lecciones sea una percepción incidida, también, por el trato de cada docente, el cual trasciende otros elementos

José Laurian Ramírez-Díaz y Fiorella Hidalgo-Solano

Los artículos de la Revista Electrónica Educare del Centro de Investigación y Docencia en Educación de la Universidad Nacional, Costa Rica, se comparten bajo términos de la Licencia Creative Commons: Reconocimiento, № Comercial, Sin Obra Derivada 3.0 Costa Rica. Las autorizaciones adicionales a las aquí delimitadas se pueden obtener en el correo: educare@una.cr 
relacionados con la motivación estudiantil. Esto podría explicar el porqué, aunque la valoración de la infraestructura y trato docente por parte del estudiantado no se encuentre en niveles óptimos, existan personas que continúen sus estudios hasta el final: porque se trata de escenarios de relaciones interpersonales, en donde diversos elementos se conjugan, en forma compleja y particular.

Aunado a lo anterior, los componentes extrínsecos de motivación parecen ser los que mayor demanda tienen por parte de la comunidad estudiantil. Las instalaciones educativas, el acceso al colegio, la iluminación y el tamaño de las aulas son elementos de suma relevancia para el proceso educativo, ya que proponen la satisfacción del sentido de pertenencia en las personas. Debe tenerse claro que los estudios demandan un tiempo específico, en el que los sujetos permanecen en un sitio para aprender por varias horas, de modo que la confortabilidad que presentan las instalaciones es un elemento de consideración importante para que un sujeto considere su permanencia o no y, tal como se ha explicado, el trato docente juega un papel de suma relevancia en este aspecto.

Con base en lo anterior, la inversión en mejora de infraestructura y el cumplimiento de los objetivos de aprendizaje, considerados en el número y cumplimiento del total de lecciones, son dos situaciones relevantes que coadyuvan la satisfacción con los procesos de estudio y, en consecuencia, la prevención del abandono escolar.

Por tanto, entre las características más relevantes de la infraestructura que motivan al estudiantado para mantenerse en las aulas están las condiciones básicas necesarias del inmueble, como pueden ser la iluminación y el espacio físico suficiente para la comodidad durante las lecciones.

El personal docente parece percibirse dentro de este marco, de modo que entre las dinámicas que incentivan la permanencia de dicentes está el cumplimiento de los objetivos de estudio, las relaciones horizontales que se dan dentro del salón de clases (recuérdese que la mayoría de estudiantes en educación nocturna son personas adultas), y parece que los estilos de comunicación entre los integrantes del salón de clase juegan un papel relevante en este sentido.

Respectoa la motivación quecada persona tiene sobre su proyecto deestudio y permanencia en el salón de clase, parece ser que además de las metas personales, el personal docente es un elemento de suma valía, el cual trasciende incluso las mismas condiciones del salón de clase. Se entiende que la construcción del proyecto de vida de cada persona es particular, pero en el proceso inciden varios componentes, como pueden ser las personas que integran los procesos respectivos, donde pueden considerarse a los compañeros y las compañeras de clase, por un lado, y al profesorado, por otro. Las relaciones interpersonales son, a juicio de quienes escriben, un elemento de mucha valía para considerar intervenciones preventivas del abandono escolar, ya que pueden marcar la diferencia entre una decisión de continuar con los estudios, o desertar.

En resumen, invertir en el establecimiento de buenas relaciones interpersonales dentro del salón de clase, considerando a todos los agentes educativos (relaciones entre docentes y estudiantes y demás personal de la institución educativa), donde el profesorado ejerza un 
liderazgo que motive a la consecución de las metas formativas, es una estrategia que parece tener mucha aceptación por el estudiantado en contextos nocturnos y, aparentemente, de alta eficacia en estrategias de prevención del abandono de las aulas por parte de estos grupos de estudiantes.

\section{Referencias}

Abarca, S., Cáceres, S., Jiménez, E., Moraleda, V.y Romero B. (2013). Satisfacción de los alumnos con la institución universitaria y el rendimiento académico. Revista Electrónica de Investigación Docencia Creativa, 2, 48-53. Recuperado de http://hdl.handle.net/10481/27613

Baracho, A. S. (2010). La relación entre motivación y aprendizaje en el E/LE. Revista Litteris-Lingüística, 5, 1-10. Recuperado de https://www.yumpu.com/es/document/view/28876732/la-relacianentre-motivacian-y-aprendizaje-en-el-e-le

Bertoglia, L. (2005). La interacción profesor-alumno. Una visión desde los procesos atribucionales. Psicoperspectivas, 4(1), 57-73. Recuperado de http://psicoperspectivas.cl/index.php/ psicoperspectivas/article/view/26/26

Brea, L. M. (2014). Factores determinantes del sentido de pertenencia de los estudiantes de arquitectura de la Pontificia Universidad Católica Madre y Maestra, Campus Santo Tomás de Aquino (Tesis de doctorado). Universidad de Murcia, Murcia, España. Recuperado de http://www.tdx.cat/bitstream/handle/10803/284952/TLMBA.pdf?sequence =1

Cardona, D. y Agudelo, H. B. (2007). Satisfacción personal como componente de la calidad de vida de los adultos de Medellín. Revista de Salud Pública, 9(4), 541-549. doi: https://doi. org/10.1590/S0124-00642007000400006

Casas, F. (1996). Bienestar social. Una introducción psicosociológica. Barcelona: PPU.

Edel, R. (2003). Factores asociados al rendimiento académico. Revista Iberoamericana de Educación, 1-21. Recuperado de http://rieoei.org/investigacion.htm

Fallas, H.y Ross, A. (26 de mayo, 2014). 142 colegios redujeron fuga de alumnos en 57\% entre 2011 y 2013. Mística de docentes salvó a 6.000 colegiales de desertar. La Nación. Recuperado de http://www.nacion.com/gnfactory/especiales/2014/desercionestudiantil/

Flores, I. (2005). Identidad cultural y el sentimiento de pertenencia a un espacio social: Una discusión teórica. La Palabra y el Hombre, 136, 41-48. Recuperado de http://cdigital.uv.mx/ bitstream/123456789/345/1/2005136P41.pdf

Garbanzo, G. M. (2007). Factores asociados al rendimiento académico en estudiantes universitarios, una reflexión desde la calidad de la educación superior pública. Revista Educación, 31(1), 43-63. doi: https://doi.org/10.15517/revedu.v31i1.1252 
Gellnier, O. (1967). El secreto de las instituciones competitivas. España: Ediciones TEA.

González-Pienda, J. A. (2003). El rendimiento escolar. Un análisis de las variables que lo condicionan. Revista Galego-Portuguesa de Psicoloxía e Educación, 8(7), 247-258. Recuperado de http://ruc.udc.es/dspace/bitstream/handle/2183/6952/RGP 9-17. pdf? sequence $=1$ \&isAllowed $=y$

Gutiérrez, M. y Gonçalves, T.-O. (2013). Activos para el desarrollo, ajuste y bienestar subjetivo de los adolescentes. International Journal of Psychology and Psychological Therapy, 13(3), 339-355. Recuperado de http://www.ijpsy.com/volumen13/num3/366/ajuste-escolar-ybienestar-en-adolescentes-ES.pdf

Herrera, L. (2012). Principales causas de la deserción estudiantil y técnicas aplicadas para su prevención desde la gestión del Colegio Nocturno La Unión y en el Colegio Nacional Virtual Marco Tulio Salazar en el cantón de La Unión. Revista Gestión de la Educación, 2(2), 1-34. doi: https://doi.org/10.15517/rge.v2i2.5865

Jiménez, A., Terriquez, B. y Robles, F. J. (2011). Evaluación de la satisfacción académica de los estudiantes de la Universidad Autónoma de Nayarit. Revista Fuente, 3(6), 46-56. Recuperado de http://fuente.uan.edu.mx/publicaciones/02-06/8.pdf

Jiménez, W. y Gaete, M. (2010). Informe de investigación. Abandono (deserción) escolar en la enseñanza secundaria en Costa Rica, 2009-2010. San José, Costa Rica: Ministerio de Educación Pública. Recuperado de http://www.mep.go.cr/sites/default/files/DesercionSecundaria.pdf

León, F. J. (2011). Bienestar estudiantil. Significados que otorgan estudiantes y profesores (Tesis de maestría). Universidad de Chile, Santiago, Chile. Recuperado de http://repositorio.uchile. cl/tesis/uchile/2011/cs-leon f/pdfAmont/cs-leon f.pdf

Luna, F. J. (2012). Bienestar subjetivo y satisfacción escolar en la adolescencia (Tesis doctoral). Universitat de Girona, Cataluña, España. Recuperado de http://www.tesisenred.net/ bitstream/handle/10803/117735/txls.pdf?sequence $=5$

Mingorance, A. (2010). Cómo motivar al alumnado. Innovación y experiencias educativas, 30, 1-10. Recuperado de http://www.csi-csif.es/andalucia/modules/mod ense/revista/pdf/ Numero 30/AURORA MINGORANCE.pdf

Montes, I. y Lerner, J. (2011). Rendimiento académico de los estudiantes de pregrado de la Universidad EAFIT. Perspectiva cuantitativa. Colombia: Universidad de EAFIT.

Müggenburg, M. C.y Pérez, I. (2007). Tipos de estudio en el enfoque de investigación cuantitativa. Revista Enfermería Universitaria, 4(1), 35-38. Recuperado de http://www.revistas.unam.mx/ index.php/reu/article/view/30300 
Navarrete, B. (2009). La motivación en el aula. Funciones del profesor para mejorar la motivación en el aprendizaje. Innovación y experiencias educativas, 15, 1-9. Recuperado de http://www. csi-csif.es/andalucia/modules/mod ense/revista/pdf/Numero 15/BELEN NAVARRETE 1.pdf

Naranjo, M. L. (2009). Motivación: Perspectivas teóricas y algunas consideraciones de su importancia en el ámbito educativo. Revista educación, 33(2), 153-170. doi: https://doi. org/10.15517/revedu.v33i2.510

Ospina, J. (2006). La motivación, motor del aprendizaje. Revista Ciencias de la Salud, 4(Especial), 158160. Recuperado de https://revistas.urosario.edu.co/index.php/revsalud/article/view/548/472

Papalia, D. E., Duskin, R. y Martorell, G. (2012). Desarrollo humano (12a ed.). México: McGrawHill. Recuperado de https://www.academia.edu/23288132/Desarrollo Humano. Papalia $12 \mathrm{a}$ edici\%C3\%B3n

Peña, J. y Sánchez, J. (2009). Economía y felicidad: Un análisis empírico de los determinantes del bienestar subjetivo de la población. Universidad de Santiago de Compostela. Recuperado de http://www.usc.es/congresos/xiirem/pdf/46.pdf

Programa Estado de la Nación. (2015). Quinto informe del estado de la educación 2015. San José, Costa Rica: Autor. Recuperado de http://www.estadonacion.or.cr/educacion2015/index.html

Ramírez, J. (2015). Diagnóstico de necesidades estudiantiles. Cartago, Costa Rica: CTP Mario Quirós Sasso, Sección Nocturna. Manuscrito inédito.

Rojas, M. E. (2000). La deserción escolar en Costa Rica: Un estudio de causas y consecuencias en una institución educativa. Diálogos Revista Electrónica de Historia, 1(4), 1-26. Recuperado de https://revistas.ucr.ac.cr/index.php/dialogos/article/view/6352/6055

Sáenz, R. (2004). Vulnerabilidad de la infraestructura física educativa. San José, Costa Rica: Comisión Nacional de Emergencias.

Soriano, M. M. (2001). La motivación, pilar básico de todo tipo de esfuerzo. Proyecto Social: Revista de Relaciones Laborales, 9, 1-21. Recuperado de https://dialnet.unirioja.es/ revista/1070/A/2001

Teixedó, J. (2005). Los centros educativos como organizaciones. Grupo de Recerca en Organitzaó de Centres. Recuperado de http://www.joanteixido.org/doc/org-educat/centro como organizacion.pdf

Urzúa, A. y Caqueo-Urízar, A. (2012). Calidad de vida: Una revisión teórica del concepto. Terapia psicológica, 30(1), 61-71. doi: https://doi.org/10.4067/S0718-48082012000100006 